\title{
Noncommutative Sprott systems and their jerk dynamics
}

\author{
Marcin Daszkiewicz \\ Institute of Theoretical Physics \\ University of Wroclaw pl. Maxa Borna 9, 50 - 206 Wroclaw, Poland \\ e - mail : marcin@ift.uni.wroc.pl
}

\begin{abstract}
In this article we provide the noncommutative Sprott models. We demonstrate, that effectively, each of them is described by system of three complex, ordinary and nonlinear differential equations. Apart of that, we find for such modified models the corresponding (noncommutative) jerk dynamics as well as we study numerically as an example, the deformed Sprott-A system.
\end{abstract}


In the last four decades there appeared a lot of papers dealing with so-called chaotic models, i.e., with such models whose dynamics is described by strongly sensitive with respect initial conditions, nonlinear differential equations. The most popular of them are: Lorenz system [1], Roessler system [2], Rayleigh-Benard system [3, Henon-Heiles system [4, jerk equation [5], Duffing equation [6], Lotka-Volter system [7], Liu system [8], Chen system [9] and Sprott systems [10].

The quite interesting among the above models seem to be the last ones, i.e., the Sprott systems. They have been provided in article [10] by systematic examination of most simple, three-dimensional and ordinary differential equations, with quadratic nonlinearities. There has been uncovered 19 examples of chaotic flows listed in the following Table:

\begin{tabular}{cccc}
\hline type & 1st equation & 2nd equation & 3rd equation \\
\hline $\mathrm{A}$ & $\dot{x}_{1}=x_{2}$ & $\dot{x}_{2}=-x_{1}+x_{2} x_{3}$ & $\dot{x}_{3}=1-x_{2}^{2}$ \\
$\mathrm{~B}$ & $\dot{x}_{1}=x_{2} x_{3}$ & $\dot{x}_{2}=x_{1}-x_{2}$ & $\dot{x}_{3}=1-x_{1} x_{2}$ \\
$\mathrm{C}$ & $\dot{x}_{1}=x_{2} x_{3}$ & $\dot{x}_{2}=x_{1}-x_{2}$ & $\dot{x}_{3}=1-x_{1}^{2}$ \\
$\mathrm{D}$ & $\dot{x}_{1}=-x_{2}$ & $\dot{x}_{2}=x_{1}+x_{3}$ & $\dot{x}_{3}=x_{1} x_{3}+3 x_{2}^{2}$ \\
$\mathrm{E}$ & $\dot{x}_{1}=x_{2} x_{3}$ & $\dot{x}_{2}=x_{1}^{2}-x_{2}$ & $\dot{x}_{3}=1-4 x_{1}$ \\
$\mathrm{~F}$ & $\dot{x}_{1}=x_{2}+x_{3}$ & $\dot{x}_{2}=-x_{1}+0.5 x_{2}$ & $\dot{x}_{3}=x_{1}^{2}-x_{3}$ \\
$\mathrm{G}$ & $\dot{x}_{1}=0.4 x_{1}+x_{3}$ & $\dot{x}_{2}=x_{1} x_{3}-x_{2}$ & $\dot{x}_{3}=-x_{1}+x_{2}$ \\
$\mathrm{H}$ & $\dot{x}_{1}=-x_{2}+x_{3}^{2}$ & $\dot{x}_{2}=x_{1}+0.5 x_{2}$ & $\dot{x}_{3}=x_{1}-x_{3}$ \\
$\mathrm{I}$ & $\dot{x}_{1}=-0.2 x_{2}$ & $\dot{x}_{2}=x_{1}+x_{3}$ & $\dot{x}_{3}=x_{1}+x_{2}^{2}-x_{3}$ \\
$\mathrm{~J}$ & $\dot{x}_{1}=2 x_{3}$ & $\dot{x}_{2}=-2 x_{2}+x_{3}$ & $\dot{x}_{3}=-x_{1}+x_{2}+x_{2}^{2}$ \\
$\mathrm{~K}$ & $\dot{x}_{1}=x_{1} x_{2}-x_{3}$ & $\dot{x}_{2}=x_{1}-x_{2}$ & $\dot{x}_{3}=x_{1}+0.3 x_{3}$ \\
$\mathrm{~L}$ & $\dot{x}_{1}=x_{2}+3.9 x_{3}$ & $\dot{x}_{2}=0.9 x_{1}^{2}-x_{2}$ & $\dot{x}_{3}=1-x_{1}$ \\
$\mathrm{M}$ & $\dot{x}_{1}=-x_{3}$ & $\dot{x}_{2}=-x_{1}^{2}-x_{2}$ & $\dot{x}_{3}=1.7+1.7 x_{1}+x_{2}$ \\
$\mathrm{~N}$ & $\dot{x}_{1}=-2 x_{2}$ & $\dot{x}_{2}=x_{1}+x_{3}^{2}$ & $\dot{x}_{3}=1+x_{2}-2 x_{1}$ \\
$\mathrm{O}$ & $\dot{x}_{1}=x_{2}$ & $\dot{x}_{2}=x_{1}-x_{3}$ & $\dot{x}_{3}=x_{1}+x_{1} x_{3}+2.7 x_{2}$ \\
$\mathrm{P}$ & $\dot{x}_{1}=2.7 x_{2}+x_{3}$ & $\dot{x}_{2}=-x_{1}+x_{2}^{2}$ & $\dot{x}_{3}=x_{1}+x_{2}$ \\
$\mathrm{Q}$ & $\dot{x}_{1}=-x_{3}$ & $\dot{x}_{2}=x_{1}-x_{2}$ & $\dot{x}_{3}=3.1 x_{1}+x_{2}^{2}+0.5 x_{3}$ \\
$\mathrm{R}$ & $\dot{x}_{1}=0.9-x_{2}$ & $\dot{x}_{2}=0.4+x_{3}$ & $\dot{x}_{3}=x_{1} x_{2}-x_{3}$ \\
$\mathrm{~S}$ & $\dot{x}_{1}=-x_{1}-4 x_{2}$ & $\dot{x}_{2}=x_{1}+x_{3}^{2}$ & $\dot{x}_{3}=1+x_{1}$ \\
\hline
\end{tabular}

Table 1. The Sprott systems.

The above models have been applied, for example, in Electronics [11, Physics [12] and Chemistry [13]. Besides, the corresponding jerk dynamics were introduced in paper [14] by using of the proper nonlinear transformation rules of variables $x_{1}, x_{2}$ and $x_{3}$; they look as follows: 


\begin{tabular}{cc}
\hline type & jerk dynamics \\
\hline $\mathrm{A}$ & ---------- \\
$\mathrm{B}$ & ----------- \\
$\mathrm{C}$ & ----------- \\
$\mathrm{D}$ & $\dddot{x}_{1}=x_{1} \ddot{x}_{1}-\dot{x}_{1}-3 \dot{x}_{1}^{2}+x_{1}^{2}$ \\
$\mathrm{E}$ & ---------- \\
$\mathrm{F}$ & $\dddot{x}_{1}=-0.5 \ddot{x}_{1}-0.5 \dot{x}_{1}-0.5 x_{1}^{2}+2 \dot{x}_{1} x_{1}-x_{1}$ \\
$\mathrm{G}$ & $\dddot{x}_{1}=-0.6 \ddot{x}_{1}-0.6 \dot{x}_{1}-0.4 x_{1}^{2}+\dot{x}_{1} x_{1}-x_{1}$ \\
$\mathrm{H}$ & $\dddot{x}_{3}=-0.5 \ddot{x}_{3}-0.5 \dot{x}_{3}-0.5 x_{3}^{2}+2 \dot{x}_{3} x_{3}-x_{3}$ \\
$\mathrm{I}$ & $\dddot{x}_{1}=-\ddot{x}_{1}-0.2 \dot{x}_{1}-5 \dot{x}_{1}^{2}-0.4 x_{1}$ \\
$\mathrm{~J}$ & $\dddot{x}_{2}=-2 \ddot{x}_{2}-\dot{x}_{2}-4 x_{2}+2 \dot{x}_{2} x_{2}$ \\
$\mathrm{~K}$ & $\dddot{x}_{2}=-0.7 \ddot{x}_{2}-0.7 \dot{x}_{2}-x_{2}+x_{2} \ddot{x}_{2}+1.7 x_{2} \dot{x}_{2}-0.3 x_{2}^{2}+\dot{x}_{2}^{2}$ \\
$\mathrm{~L}$ & $\dddot{x}_{1}=-\ddot{x}_{1}-3.9 \dot{x}_{1}-3.9 x_{1}+1.8 x_{1} \dot{x}_{1}+3.9$ \\
$\mathrm{M}$ & $\dddot{x}_{1}=-\ddot{x}_{1}-1.7 \dot{x}_{1}-1.7 x_{1}+x_{1}^{2}-1.7$ \\
$\mathrm{~N}$ & $\dddot{x}_{3}=-2 \ddot{x}_{3}-2 \dot{x}_{3}-4 x_{3}+2 \dot{x}_{3} x_{3}+2$ \\
$\mathrm{O}$ & $\dddot{x}_{1}=x_{1} \ddot{x}_{1}-1.7 \dot{x}_{1}-x_{1}-x_{1}^{2}$ \\
$\mathrm{P}$ & $\dddot{x}_{2}=2 x_{2} \ddot{x}_{2}-1.7 \dot{x}_{2}+2 \dot{x}_{2}^{2}-x_{2}-x_{2}^{2}$ \\
$\mathrm{Q}$ & $\dddot{x}_{2}=-0.5 \ddot{x}_{2}-2.6 \dot{x}_{2}-3.1 x_{2}-x_{2}^{2}$ \\
$\mathrm{R}$ & $\dddot{x}_{1}=-\ddot{x}_{1}-0.9 x_{1}+x_{1} \dot{x}_{1}-0.4$ \\
$\mathrm{~S}$ & $\dddot{x}_{3}=-\ddot{x}_{3}-4 \dot{x}_{3}-4 x_{3}^{2}+4$ \\
\hline
\end{tabular}

Table 2a. The jerk dynamics.

\begin{tabular}{|c|c|c|}
\hline type & \multicolumn{2}{|c|}{ remaining trajectories } \\
\hline A & \multicolumn{2}{|c|}{----------} \\
\hline $\mathrm{B}$ & \multicolumn{2}{|c|}{----------} \\
\hline $\mathrm{C}$ & \multicolumn{2}{|c|}{---------} \\
\hline $\mathrm{D}$ & $x_{2}=-\dot{x}_{1}$ & $x_{3}=-\ddot{x}_{1}-x_{1}$ \\
\hline $\mathrm{E}$ & \multicolumn{2}{|c|}{----------} \\
\hline $\mathrm{F}$ & $x_{2}=0.6\left(\ddot{x}_{1}+\dot{x}_{1}+x_{1}-x_{1}^{2}\right)$ & $x_{3}=0.6\left(-\ddot{x}_{1}+0.5 \dot{x}_{1}-x_{1}+x_{1}^{2}\right)$ \\
\hline $\mathrm{G}$ & $x_{2}=\ddot{x}_{1}-0.4 \dot{x}_{1}+x_{1}$ & $x_{3}=\dot{x}_{1}-0.4 x_{1}$ \\
\hline $\mathrm{H}$ & $x_{1}=\dot{x}_{3}+x_{3}$ & $x_{2}=-\ddot{x}_{3}-\dot{x}_{3}+x_{3}^{2}$ \\
\hline I & $x_{2}=-5 \dot{x}_{1}$ & $x_{3}=-5 \ddot{x}_{1}-x_{1}$ \\
\hline $\mathrm{J}$ & $x_{1}=-\ddot{x}_{2}-2 \dot{x}_{2}+x_{2}+x_{2}^{2}$ & $x_{3}=2 x_{2}+\dot{x}_{2}$ \\
\hline $\mathrm{K}$ & $x_{1}=\dot{x}_{2}+x_{2}$ & $x_{3}=-\ddot{x}_{2}-\dot{x}_{2}+x_{2} \dot{x}_{2}+x_{2}^{2}$ \\
\hline $\mathrm{L}$ & $x_{2}=-\ddot{x}_{1}-3.9 x_{1}+0.9 x_{1}^{2}+3.9$ & $\begin{aligned} x_{3}= & 0.2\left(\ddot{x}_{1}+\dot{x}_{1}+3.9 x_{1}+\right. \\
& \left.-0.9 x_{1}^{2}-3.9\right)\end{aligned}$ \\
\hline M & $x_{2}=-\ddot{x}_{1}-1.7 x_{1}-1.7$ & $x_{3}=-\dot{x}_{1}$ \\
\hline $\mathrm{N}$ & $x_{1}=\ddot{x}_{3}+2 \dot{x}_{3}-x_{3}^{2}$ & $x_{2}=\dot{x}_{3}+2 x_{3}-1$ \\
\hline $\mathrm{O}$ & $x_{2}=\dot{x}_{1}$ & $x_{3}=-\ddot{x}_{1}+x_{1}$ \\
\hline $\mathrm{P}$ & $x_{1}=-\dot{x}_{2}+x_{2}^{2}$ & $x_{3}=-\ddot{x}_{2}+2 x_{2} \dot{x}_{2}-2.7 x_{2}$ \\
\hline Q & $x_{1}=\dot{x}_{2}+x_{2}$ & $x_{3}=-\ddot{x}_{2}-\dot{x}_{2}$ \\
\hline $\mathrm{R}$ & $x_{2}=-\dot{x}_{1}+0.9$ & $x_{3}=-\ddot{x}_{1}-0.4$ \\
\hline $\mathrm{S}$ & $x_{1}=\dot{x}_{3}-1$ & $x_{2}=0.25\left(-\ddot{x}_{3}-\dot{x}_{3}+1\right)$ \\
\hline
\end{tabular}


Table 2b. The jerk dynamics - remaining trajectories.

In this article we define the Sprott systems on noncommutative space of the form

$$
\left[\hat{x}_{i}, \hat{x}_{j}\right]=i f_{i j}(t)=i f(t) \epsilon_{i j}
$$

with arbitrary time-dependent real functions $f(t)$ and antisymmetric tensor $\epsilon_{i j}=-\epsilon_{j} \bigsqcup^{1}$. The motivations for such studies are quite obvious and natural, i.e., it gives the general information on the impact of high-energy (just noncommutative) regime on the one of the most popular chaotic (nonlinear) models. It should be noted, however, that such kind of investigations has been already performed for example in articles [19] and [20].

In the first step of our construction we remind, that the commutation relationes (1) can be (formally) realized in the framework of Quantum Group Theory [21], with use so-called twist procedure [22]. Then, the quantum space is represented by Hopf module equipped with the following $\star$-product for two arbitrary classical functions $f(x)$ and $g(x)$ 23

$$
f(x) \star g(y)=f(x) \exp \left(\frac{i}{2} f_{i j}(t) \partial_{x}^{i} \otimes \partial_{y}^{j}\right) g(y)
$$

Particularly, for $f(x)=x$ and $g(y)=y$ we have

$$
\begin{aligned}
x_{i} \star x_{j} & =x_{i} \exp \left(\frac{i}{2} f_{k l}(t) \partial_{x}^{k} \otimes \partial_{x}^{l}\right) x_{j}=x_{i} x_{j}+i \frac{f_{i j}(t)}{2}, \\
x_{j} \star x_{i} & =x_{j} \exp \left(\frac{i}{2} f_{k l}(t) \partial_{x}^{k} \otimes \partial_{x}^{l}\right) x_{i}=x_{j} x_{i}+i \frac{f_{j i}(t)}{2},
\end{aligned}
$$

and, consequently

$$
\left[\hat{x}_{i}, \hat{x}_{j}\right]=\left[x_{i}, x_{j}\right]_{\star}=x_{i} \star x_{j}-x_{j} \star x_{i}=i f_{i j}(t) .
$$

It should be noted, that quantum space (1) has been explicitly constructed only for particular form of function $f_{i j}(t)$ in papers [23], 24] and [25].

Next, we define the noncommutative Sprott systems by the following replacement

$$
x_{i} x_{j} \rightarrow x_{i} \star x_{j}=x_{i} x_{j}+i \frac{f_{i j}(t)}{2} ; i \neq j
$$

provided in all dynamical equations from Table 1. In such a way, we get:

\footnotetext{
${ }^{1}$ One should notice, that the commutation relations $(1)$ reproduce for constant function $f_{i j}(t)=\theta_{i j}$ the well-known, most simple version of deformed space, called canonical type of space-time noncommutativity [15- 18 .
} 


\begin{tabular}{cccc}
\hline type & 1st equation & 2nd equation & 3rd equation \\
\hline $\mathrm{A}$ & $\dot{x}_{1}=x_{2}$ & $\dot{x}_{2}=-x_{1}+x_{2} \star x_{3}$ & $\dot{x}_{3}=1-x_{2}^{2}$ \\
$\mathrm{~B}$ & $\dot{x}_{1}=x_{2} \star x_{3}$ & $\dot{x}_{2}=x_{1}-x_{2}$ & $\dot{x}_{3}=1-x_{1} \star x_{2}$ \\
$\mathrm{C}$ & $\dot{x}_{1}=x_{2} \star x_{3}$ & $\dot{x}_{2}=x_{1}-x_{2}$ & $\dot{x}_{3}=1-x_{1}^{2}$ \\
$\mathrm{D}$ & $\dot{x}_{1}=-x_{2}$ & $\dot{x}_{2}=x_{1}+x_{3}$ & $\dot{x}_{3}=x_{1} \star x_{3}+3 x_{2}^{2}$ \\
$\mathrm{E}$ & $\dot{x}_{1}=x_{2} \star x_{3}$ & $\dot{x}_{2}=x_{1}^{2}-x_{2}$ & $\dot{x}_{3}=1-4 x_{1}$ \\
$\mathrm{~F}$ & $\dot{x}_{1}=x_{2}+x_{3}$ & $\dot{x}_{2}=-x_{1}+0.5 x_{2}$ & $\dot{x}_{3}=x_{1}^{2}-x_{3}$ \\
$\mathrm{G}$ & $\dot{x}_{1}=0.4 x_{1}+x_{3}$ & $\dot{x}_{2}=x_{1} \star x_{3}-x_{2}$ & $\dot{x}_{3}=-x_{1}+x_{2}$ \\
$\mathrm{H}$ & $\dot{x}_{1}=-x_{2}+x_{3}^{2}$ & $\dot{x}_{2}=x_{1}+0.5 x_{2}$ & $\dot{x}_{3}=x_{1}-x_{3}$ \\
$\mathrm{I}$ & $\dot{x}_{1}=-0.2 x_{2}$ & $\dot{x}_{2}=x_{1}+x_{3}$ & $\dot{x}_{3}=x_{1}+x_{2}^{2}-x_{3}$ \\
$\mathrm{~J}$ & $\dot{x}_{1}=2 x_{3}$ & $\dot{x}_{2}=-2 x_{2}+x_{3}$ & $\dot{x}_{3}=-x_{1}+x_{2}+x_{2}^{2}$ \\
$\mathrm{~K}$ & $\dot{x}_{1}=x_{1} \star x_{2}-x_{3}$ & $\dot{x}_{2}=x_{1}-x_{2}$ & $\dot{x}_{3}=x_{1}+0.3 x_{3}$ \\
$\mathrm{~L}$ & $\dot{x}_{1}=x_{2}+3.9 x_{3}$ & $\dot{x}_{2}=0.9 x_{1}^{2}-x_{2}$ & $\dot{x}_{3}=1-x_{1}$ \\
$\mathrm{M}$ & $\dot{x}_{1}=-x_{3}$ & $\dot{x}_{2}=-x_{1}^{2}-x_{2}$ & $\dot{x}_{3}=1.7+1.7 x_{1}+x_{2}$ \\
$\mathrm{~N}$ & $\dot{x}_{1}=-2 x_{2}$ & $\dot{x}_{2}=x_{1}+x_{3}^{2}$ & $\dot{x}_{3}=1+x_{2}-2 x_{1}$ \\
$\mathrm{O}$ & $\dot{x}_{1}=x_{2}$ & $\dot{x}_{2}=x_{1}-x_{3}$ & $\dot{x}_{3}=x_{1}+x_{1} \star x_{3}+2.7 x_{2}$ \\
$\mathrm{P}$ & $\dot{x}_{1}=2.7 x_{2}+x_{3}$ & $\dot{x}_{2}=-x_{1}+x_{2}^{2}$ & $\dot{x}_{3}=x_{1}+x_{2}$ \\
$\mathrm{Q}$ & $\dot{x}_{1}=-x_{3}$ & $\dot{x}_{2}=x_{1}-x_{2}$ & $\dot{x}_{3}=3.1 x_{1}+x_{2}^{2}+0.5 x_{3}$ \\
$\mathrm{R}$ & $\dot{x}_{1}=0.9-x_{2}$ & $\dot{x}_{2}=0.4+x_{3}$ & $\dot{x}_{3}=x_{1} \star x_{2}-x_{3}$ \\
$\mathrm{~S}$ & $\dot{x}_{1}=-x_{1}-4 x_{2}$ & $\dot{x}_{2}=x_{1}+x_{3}^{2}$ & $\dot{x}_{3}=1+x_{1}$ \\
\hline
\end{tabular}

Table 3. The noncommutative Sprott systems.

We see, that due to the formulas (3) and (4), the above models (apart of Sprott-F, H, I, J, L, M, N, P, Q and S systems) become complex; let us selectively examine them. In this aim we take under considerations the most simple (canonical) type of space noncommutativity (1) given by $f_{i j}(t)=\theta_{i j}=$ const. Then, there appears in all modified Sprott models the additional constant and pure imaginary term $\frac{i}{2} \theta_{i j}$. Below, we derive exemplary $3 \mathrm{D}$ and in $x_{1}$-direction trajectories as well as Poincaré sections for deformed Sprott-A system with parameter $\theta_{23}=1$, and with real initial conditions: $\left(x_{1}(0), x_{2}(0), x_{3}(0)\right)=(0,5,0)$, $\left(x_{1}(0), x_{2}(0), x_{3}(0)\right)=\left(0,5+\epsilon_{1}, 0\right)$ and $\left(x_{1}(0), x_{2}(0), x_{3}(0)\right)=\left(0,5+\epsilon_{2}, 0\right)$ for $\epsilon_{1}=0.00001$ and $\epsilon_{2}=0.000001$ respectively ${ }^{2}$. The obtained results are presented on Figures $\mathbf{1}-\mathbf{7}$ and they indicate, that the model remains sensitive with respect already small change of initial data. Besides, the shape of all three-dimensional trajectories as well as the form of the Poincaré sections suggest, that the investigated system irregularly oscillates along the stretch of the real $x_{1}$-axis 3 .

Further, we find by tedious calculations the corresponding (noncommutative) jerk dynamics listed in the following two Tables:

\footnotetext{
${ }^{2}$ Trajectories are given by real component of the complex solution of differential Sprott equations, i.e., it looks as follows: $\left(\operatorname{Re}\left(x_{1}(t)\right), \operatorname{Re}\left(x_{2}(t)\right), \operatorname{Re}\left(x_{3}(t)\right)\right)$.

${ }^{3}$ The numerical studies have been performed on the Mathematica program version 10.1, with the accuracy and precision both equal to 16 .
} 


\begin{tabular}{cc}
\hline type & jerk dynamics \\
\hline $\mathrm{A}$ & ---------- \\
$\mathrm{B}$ & ----------- \\
$\mathrm{C}$ & ---------- \\
$\mathrm{D}$ & $\dddot{x}_{1}=x_{1} \ddot{x}_{1}-\dot{x}_{1}-3 \dot{x}_{1}^{2}+x_{1}^{2}-\frac{i f_{13}(t)}{2}$ \\
$\mathrm{E}$ & ----------- \\
$\mathrm{F}$ & $\dddot{x}_{1}=-0.5 \ddot{x}_{1}-0.5 \dot{x}_{1}-0.5 x_{1}^{2}+2 \dot{x}_{1} x_{1}-x_{1}$ \\
$\mathrm{G}$ & $\dddot{x}_{1}=-0.6 \ddot{x}_{1}-0.6 \dot{x}_{1}-0.4 x_{1}^{2}+\dot{x}_{1} x_{1}-x_{1}+\frac{i f_{13}(t)}{2}$ \\
$\mathrm{H}$ & $\dddot{x}_{3}=-0.5 \ddot{x}_{3}-0.5 \dot{x}_{3}-0.5 x_{3}^{2}+2 \dot{x}_{3} x_{3}-x_{3}$ \\
$\mathrm{I}$ & $\dddot{x}_{1}=-\ddot{x}_{1}-0.2 \dot{x}_{1}-5 \dot{x}_{1}^{2}-0.4 x_{1}$ \\
$\mathrm{~J}$ & $\dddot{x}_{2}=-2 \ddot{x}_{2}-\dot{x}_{2}-4 x_{2}+2 \dot{x}_{2} x_{2}$ \\
$\mathrm{~K}$ & $\ddot{x}_{2}=-0.7 \ddot{x}_{2}-0.7 \dot{x}_{2}-x_{2}+x_{2} \ddot{x}_{2}+1.7 x_{2} \dot{x}_{2}-0.3 x_{2}^{2}+\dot{x}_{2}^{2}+$ \\
& $\dddot{x}_{1}=-\frac{i}{2}\left(\ddot{f}_{12}(t)-0.3 f_{12}(t)\right)$ \\
$\mathrm{L}$ & $\dddot{x}_{1}=-3.9 \dot{x}_{1}-3.9 x_{1}+1.8 x_{1} \dot{x}_{1}+3.9$ \\
$\mathrm{M}$ & $\dddot{x}_{3}=-2.7 \ddot{x}_{1}-1.7 x_{1}+x_{1}^{2}-1.7$ \\
$\mathrm{~N}$ & $\dddot{x}_{1}=x_{1} \ddot{x}_{1}-1.7 \dot{x}_{1}-4 x_{1}-x_{1}^{2}-\frac{i f_{13}(t)}{2}$ \\
$\mathrm{O}$ & $\dddot{x}_{2}=2 x_{2} \ddot{x}_{2}-1.7 \dot{x}_{2}+2 \dot{x}_{2}^{2}-x_{2}-x_{2}^{2}$ \\
$\mathrm{P}$ & $\dddot{x}_{2}=-0.5 \ddot{x}_{2}-2.6 \dot{x}_{2}-3.1 x_{2}-x_{2}^{2}$ \\
$\mathrm{Q}$ & $\dddot{x}_{1}=-\ddot{x}_{1}-0.9 x_{1}+x_{1} \dot{x}_{1}-0.4-\frac{i f_{12}(t)}{2}$ \\
$\mathrm{R}$ & $\dddot{x}_{3}=-\ddot{x}_{3}-4 \dot{x}_{3}-4 x_{3}^{2}+4$ \\
$\mathrm{~S}$ &
\end{tabular}

Table 4a. The noncommutative jerk dynamics.

\begin{tabular}{|c|c|c|}
\hline type & \multicolumn{2}{|c|}{ remaining trajectories } \\
\hline $\mathrm{A}$ & \multicolumn{2}{|c|}{----------} \\
\hline $\mathrm{B}$ & \multicolumn{2}{|c|}{----------} \\
\hline $\mathrm{C}$ & \multicolumn{2}{|c|}{----------} \\
\hline $\mathrm{D}$ & $x_{2}=-\dot{x}_{1}$ & $x_{3}=-\ddot{x}_{1}-x_{1}$ \\
\hline $\mathrm{E}$ & \multicolumn{2}{|c|}{----------} \\
\hline $\mathrm{F}$ & $x_{2}=0.6\left(\ddot{x}_{1}+\dot{x}_{1}+x_{1}-x_{1}^{2}\right)$ & $x_{3}=0.6\left(-\ddot{x}_{1}+0.5 \dot{x}_{1}-x_{1}+x_{1}^{2}\right)$ \\
\hline G & $x_{2}=\ddot{x}_{1}-0.4 \dot{x}_{1}+x_{1}$ & $x_{3}=\dot{x}_{1}-0.4 x_{1}$ \\
\hline $\mathrm{H}$ & $x_{1}=\dot{x}_{3}+x_{3}$ & $x_{2}=-\ddot{x}_{3}-\dot{x}_{3}+x_{3}^{2}$ \\
\hline $\mathrm{I}$ & $x_{2}=-5 \dot{x}_{1}$ & $x_{3}=-5 \ddot{x}_{1}-x_{1}$ \\
\hline $\mathrm{J}$ & $x_{1}=-\ddot{x}_{2}-2 \dot{x}_{2}+x_{2}+x_{2}^{2}$ & $x_{3}=2 x_{2}+\dot{x}_{2}$ \\
\hline $\mathrm{K}$ & $x_{1}=\dot{x}_{2}+x_{2}$ & $x_{3}=-\ddot{x}_{2}-\dot{x}_{2}+x_{2} \dot{x}_{2}+x_{2}^{2}+\frac{i f_{12}(t)}{2}$ \\
\hline $\mathrm{L}$ & $x_{2}=-\ddot{x}_{1}-3.9 x_{1}+0.9 x_{1}^{2}+3.9$ & $\begin{aligned} x_{3}= & 0.2\left(\ddot{x}_{1}+\dot{x}_{1}+3.9 x_{1}+\right. \\
& \left.-0.9 x_{1}^{2}-3.9\right)\end{aligned}$ \\
\hline M & $x_{2}=-\ddot{x}_{1}-1.7 x_{1}-1.7$ & $x_{3}=-\dot{x}_{1}$ \\
\hline $\mathrm{N}$ & $x_{1}=\ddot{x}_{3}+2 \dot{x}_{3}-x_{3}^{2}$ & $x_{2}=\dot{x}_{3}+2 x_{3}-1$ \\
\hline
\end{tabular}




\begin{tabular}{lcc}
$\mathrm{O}$ & $x_{2}=\dot{x}_{1}$ & $x_{3}=-\ddot{x}_{1}+x_{1}$ \\
$\mathrm{P}$ & $x_{1}=-\dot{x}_{2}+x_{2}^{2}$ & $x_{3}=-\ddot{x}_{2}+2 x_{2} \dot{x}_{2}-2.7 x_{2}$ \\
$\mathrm{Q}$ & $x_{1}=\dot{x}_{2}+x_{2}$ & $x_{3}=-\ddot{x}_{2}-\dot{x}_{2}$ \\
$\mathrm{R}$ & $x_{2}=-\dot{x}_{1}+0.9$ & $x_{3}=-\ddot{x}_{1}-0.4$ \\
$\mathrm{~S}$ & $x_{1}=\dot{x}_{3}-1$ & $x_{2}=0.25\left(-\ddot{x}_{3}-\dot{x}_{3}+1\right)$ \\
\hline
\end{tabular}

Table 4 b. The noncommutative jerk dynamics - remaining trajectories.

Consequently, in such a way, we easily observe that all founded above complex equations include additional pure imaginary terms $\frac{i}{2} f_{i j}(t)$ and $\frac{i}{2} \dot{f}_{i j}(t)$ respectively, and just for this reason, in the case of functions $f_{i j}(t)$ equal zero, the discussed models become classical.

At the end stage of this paper, it should be also mentioned, that the physical meaning of all complex components $\operatorname{Im}\left(x_{i}(t)\right)$ remains unclear. However, one may expect for example, that they play a role of additionally, generated by space-time noncommutativity, internal degrees of freedom. They are not observed or directly measured, but in spite of that, they effectively affect on the real part of the solution of differential equation.

\section{References}

[1] E.N. Lorenz, J. Atmos. Sci. 20, 130 (1963)

[2] O.E. Roessler, Phys. Lett. A 57, 397 (1976)

[3] A.V. Getling, "Rayleigh-Benard Convection: Structures and Dynamics", World Scientific, 1998

[4] M. Henon, C. Heiles, AJ. 69, 73 (1964)

[5] J.C. Sprott, Am J Phys. 65, 537 (1997)

[6] G. Duffinng, "Erzwungene Schwingungen bei Vernderlicher Eigenfrequenz", F. Vieweg u. Sohn, Braunschweig, 1918.

[7] V. Volterra, "Variations and fluctuations of the number of indviduals in animal species living together. In Animal Ecology" McGraw-Hill, 1931. Translated from 1928 edition by R. N. Chapman.

[8] C. Liu, T. Liu, L. Liu, K. Liu, Chaos, Solitons and Fractals 22, 1031 (2004)

[9] G. Chen, T. Ueta, Journal of Bifurcation and Chaos 9, 1465 (1999)

[10] J.C. Sprott, Phys. Rev. E 50, 647 (1994)

[11] J. Petrzela, Z. Hrubos, T. Gotthans, Radioengineering 20, 438 (2011) 
[12] J.C. Sprott, "Chaos and Time-Series Analysis", Oxford University Press, 2003

[13] S. Vaidyanathan and Ch. Volos (Editors), "Advances and Applications in Chaotic Systems", Springer, 2016

[14] R. Eichhorn, S.J. Linz, P. Hanggi, Phys. Rev. E 58, 7151 (1998)

[15] R. Oeckl, J. Math. Phys. 40, 3588 (1999)

[16] M.R. Douglas, N.A. Nekrasov, Rev. Mod. Phys. 73, 977 (2001)

[17] M. Szabo, Phys. Rep. 378, 207 (2003)

[18] M. Chaichian, P.P. Kulish, K. Nashijima, A. Tureanu, Phys. Lett. B 604, 98 (2004); hep-th/0408069

[19] S. Klimek, A. Lesniewski, Annals of Physics 248, 173 (1996)

[20] T. Mai, "Regularity of distributions of Wigner integrals"; arXiv: 1512.07593 [math.OA]

[21] S. Majid, "Foundations of quantum group theory", Cambridge University Press, April 2000

[22] N.Yu. Reshetikhin, Lett. Math. Phys. 20, 331 (1990)

[23] R. Oeckl, Nucl. Phys. B 581, 559 (2000)

[24] M. Daszkiewicz, Mod. Phys. Lett. A24, 1325 (2009); arXiv: 0904.0432

[25] M. Daszkiewicz, Mod. Phys. Lett. A23, 505 (2008); arXiv: 0801.1206 


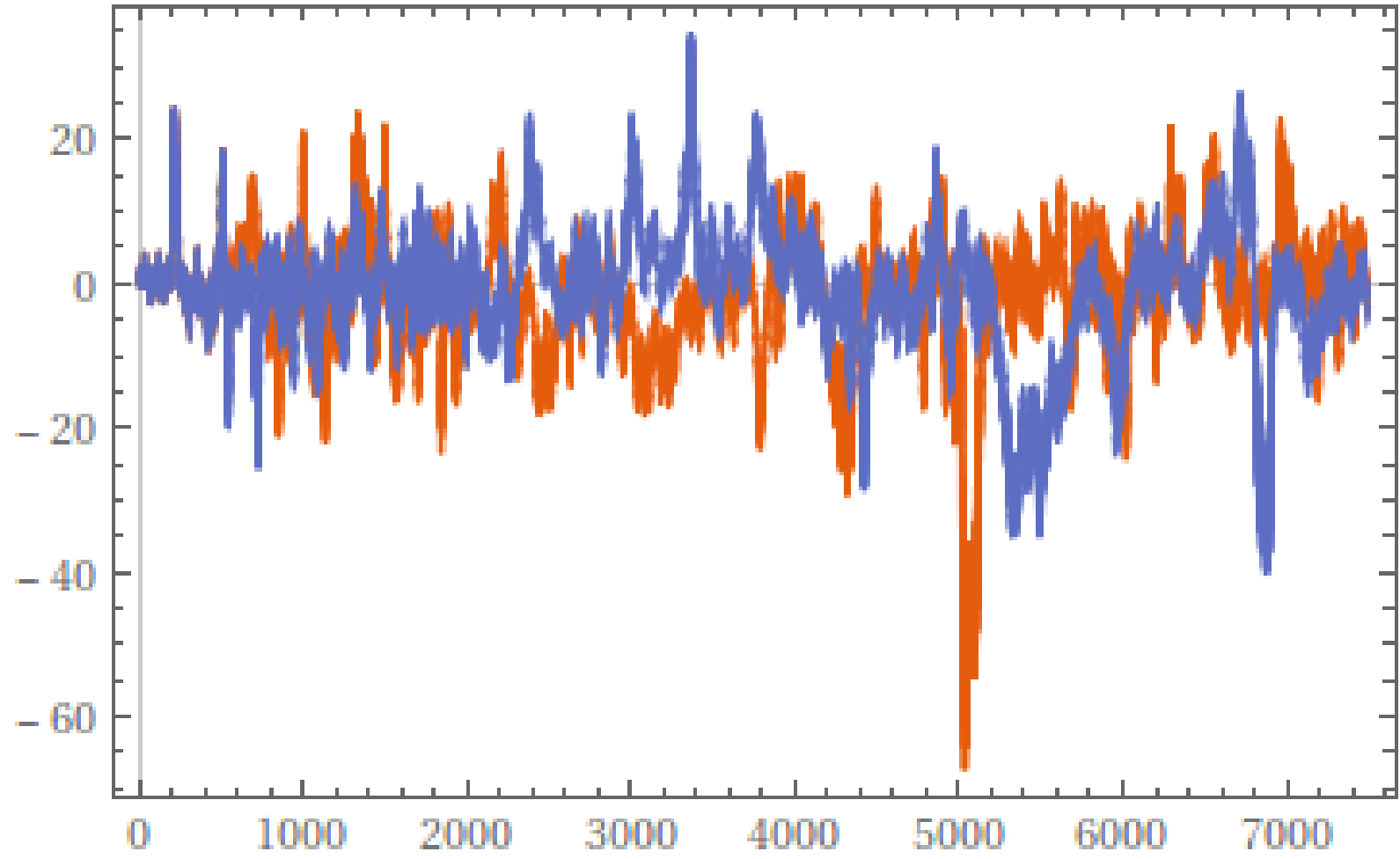

Figure 1: The $\operatorname{Re}\left(x_{1}(t)\right)$-trajectories for Sprott-A system with initial conditions $\left(x_{1}(0), x_{2}(0), x_{3}(0)\right)=(0,5,0)$ (red line) and $\left(x_{1}(0), x_{2}(0), x_{3}(0)\right)=\left(0,5+\epsilon_{1}, 0\right)$ (blue line) respectively. The time parameter runs from 0 to 7500 and the trajectories become divergent near $t$ equal to 500 . 


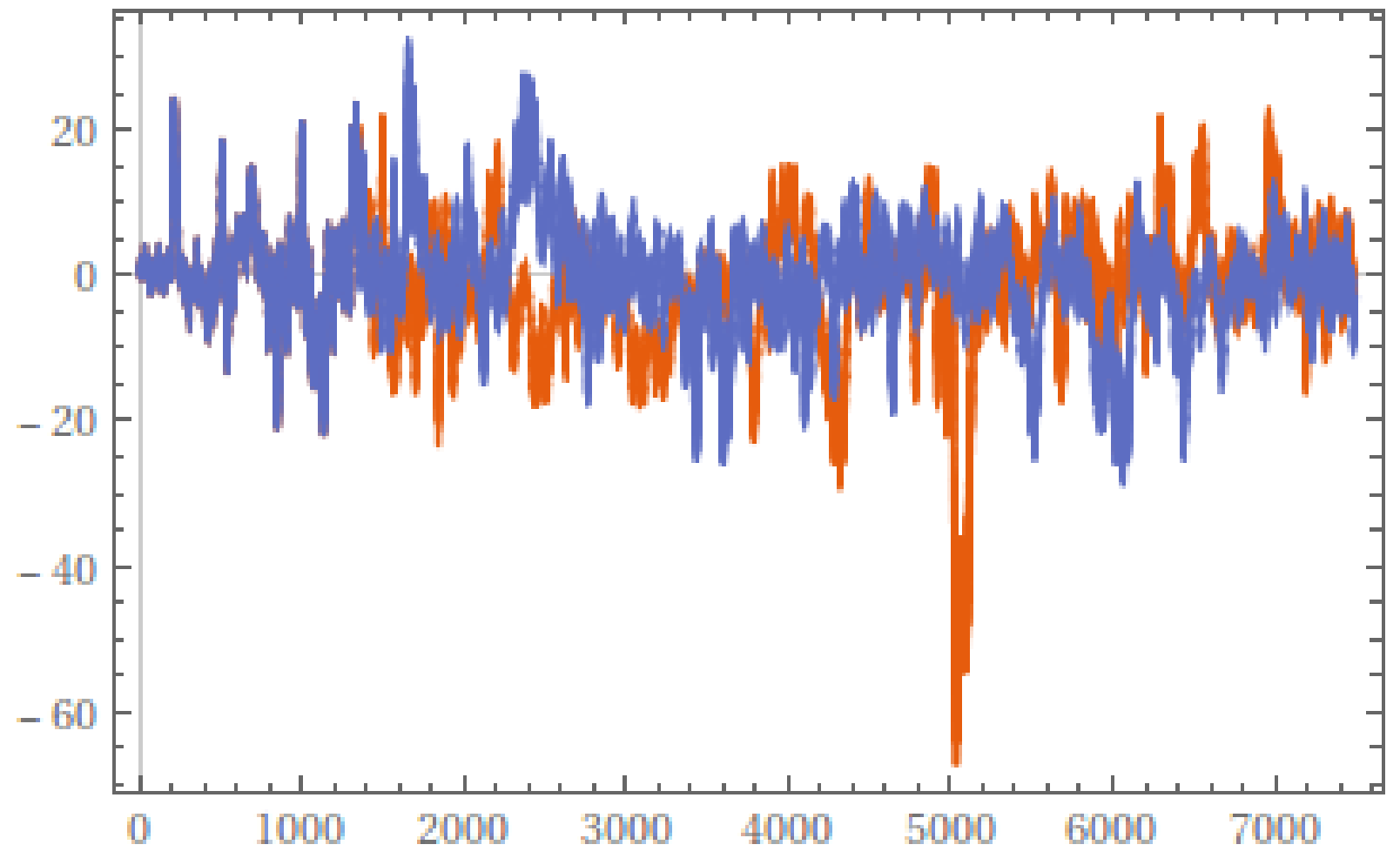

Figure 2: The $\operatorname{Re}\left(x_{1}(t)\right)$-trajectories for Sprott-A system with initial conditions $\left(x_{1}(0), x_{2}(0), x_{3}(0)\right)=(0,5,0)$ (red line) and $\left(x_{1}(0), x_{2}(0), x_{3}(0)\right)=\left(0,5+\epsilon_{2}, 0\right)$ (blue line) respectively. The time parameter runs from 0 to 7500 and the trajectories become divergent near $t$ equal to 1200 . 


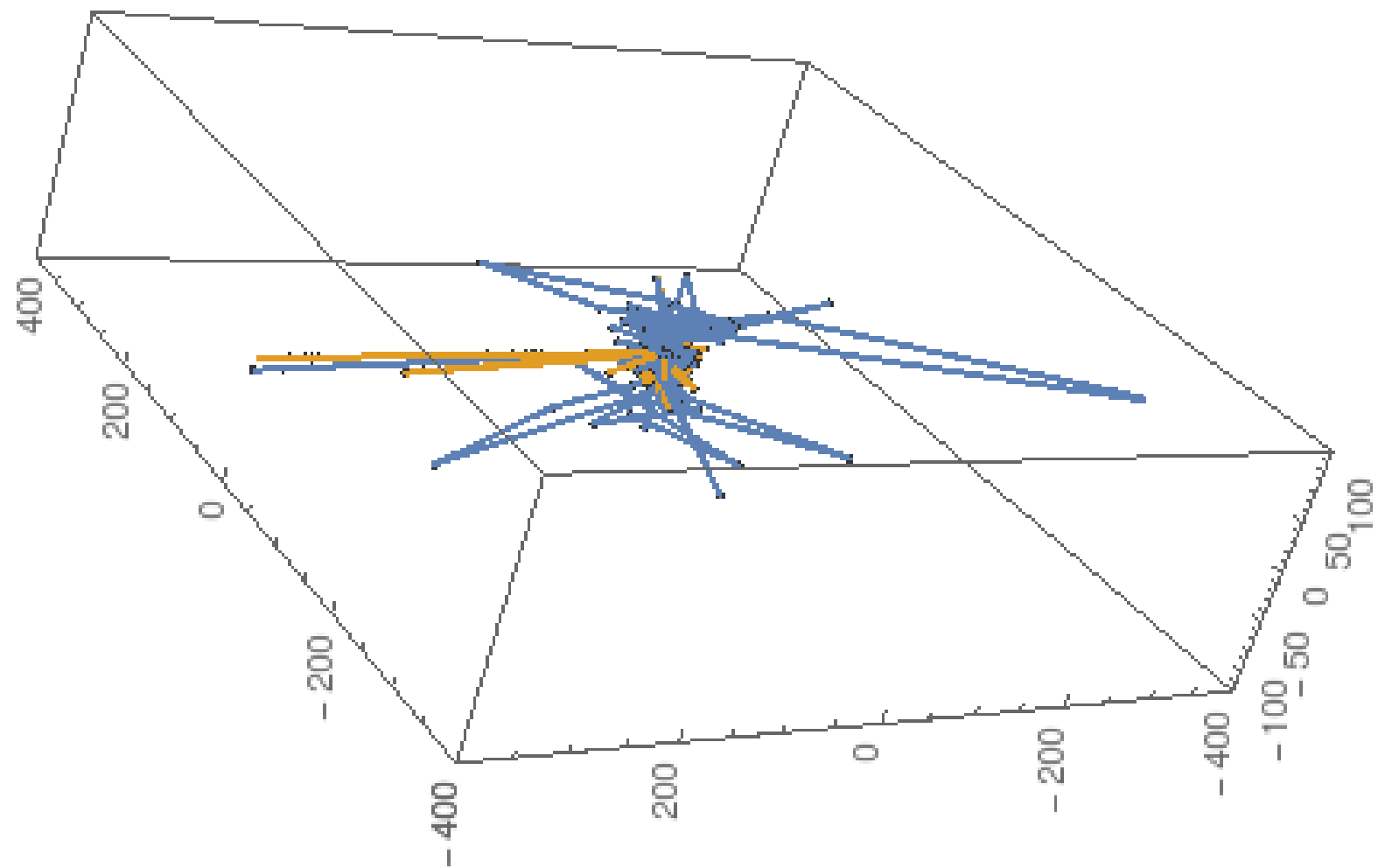

Figure 3: The 3D-trajectories for Sprott-A system with initial conditions $\left(x_{1}(0), x_{2}(0), x_{3}(0)\right)=(0,5,0)$ (blue line) and $\left(x_{1}(0), x_{2}(0), x_{3}(0)\right)=\left(0,5+\epsilon_{1}, 0\right)$ (orange line) respectively. The time parameter runs from 0 to 20000. 


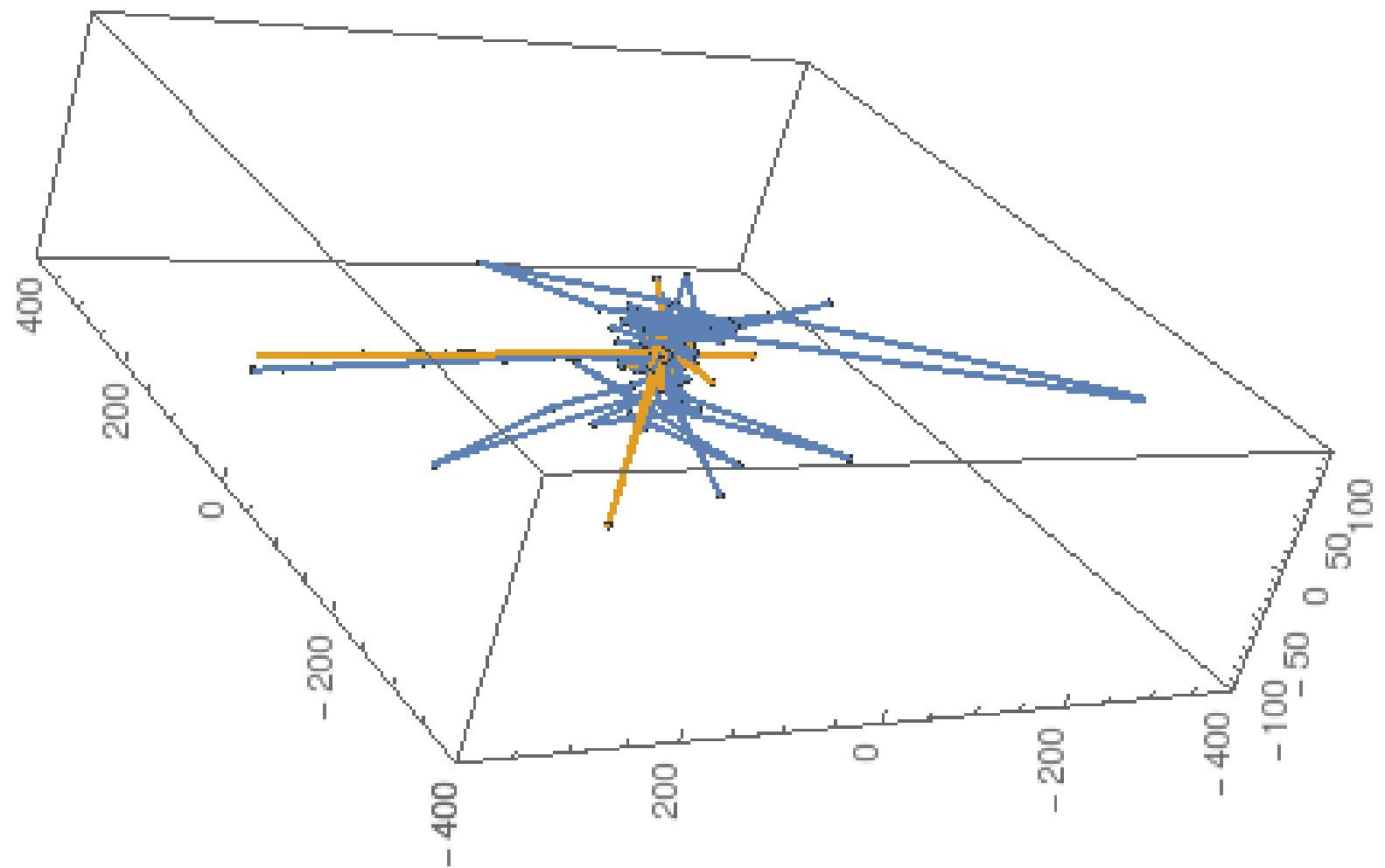

Figure 4: The 3D-trajectories for Sprott-A system with initial conditions $\left(x_{1}(0), x_{2}(0), x_{3}(0)\right)=(0,5,0)$ (blue line) and $\left(x_{1}(0), x_{2}(0), x_{3}(0)\right)=\left(0,5+\epsilon_{2}, 0\right)$ (orange line) respectively. The time parameter runs from 0 to 20000. 


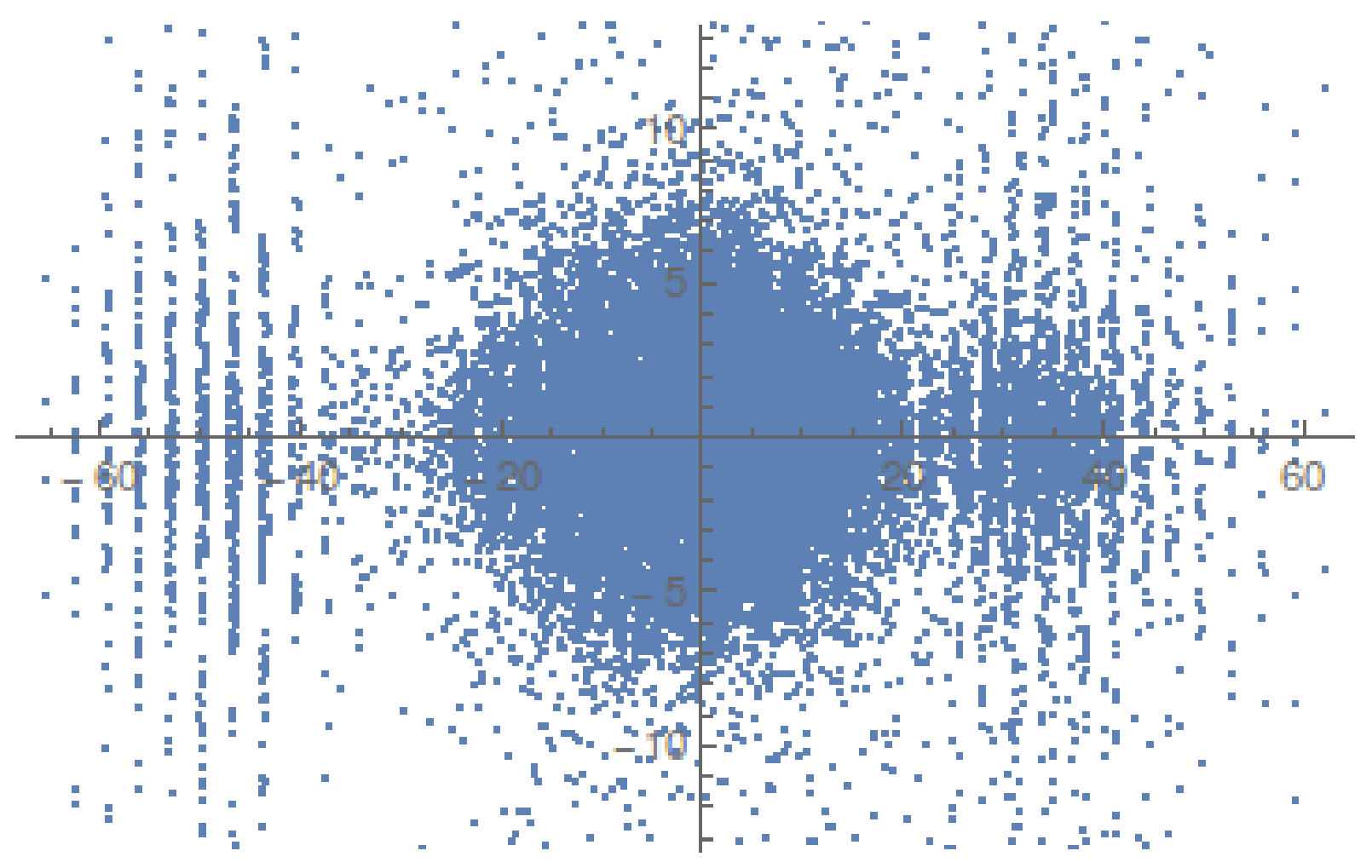

Figure 5: The Poincaré section $\left(\operatorname{Re}\left(x_{1}(t)\right), \operatorname{Re}\left(x_{3}(t)\right)\right)$ with $\operatorname{Re}\left(x_{2}(t)\right)=0$ for Sprott-A model for initial condition $\left(x_{1}(0), x_{2}(0), x_{3}(0)\right)=(0,5,0)$. The time parameter runs from 0 to 20000 . 


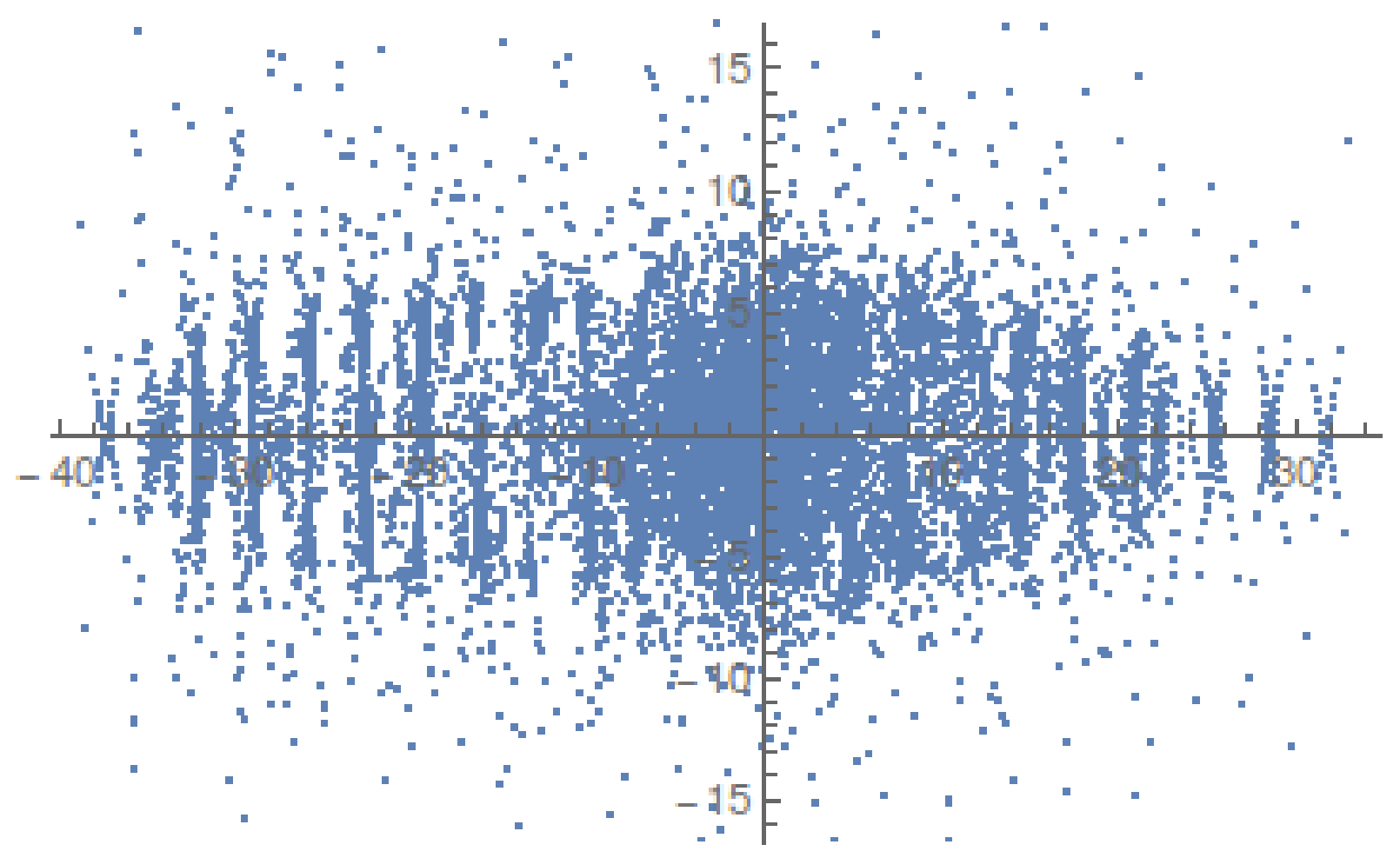

Figure 6: The Poincaré section $\left(\operatorname{Re}\left(x_{1}(t)\right), \operatorname{Re}\left(x_{3}(t)\right)\right)$ with $\operatorname{Re}\left(x_{2}(t)\right)=0$ for Sprott-A system for initial condition $\left(x_{1}(0), x_{2}(0), x_{3}(0)\right)=\left(0,5+\epsilon_{1}, 0\right)$. The time parameter runs from 0 to 20000 . 


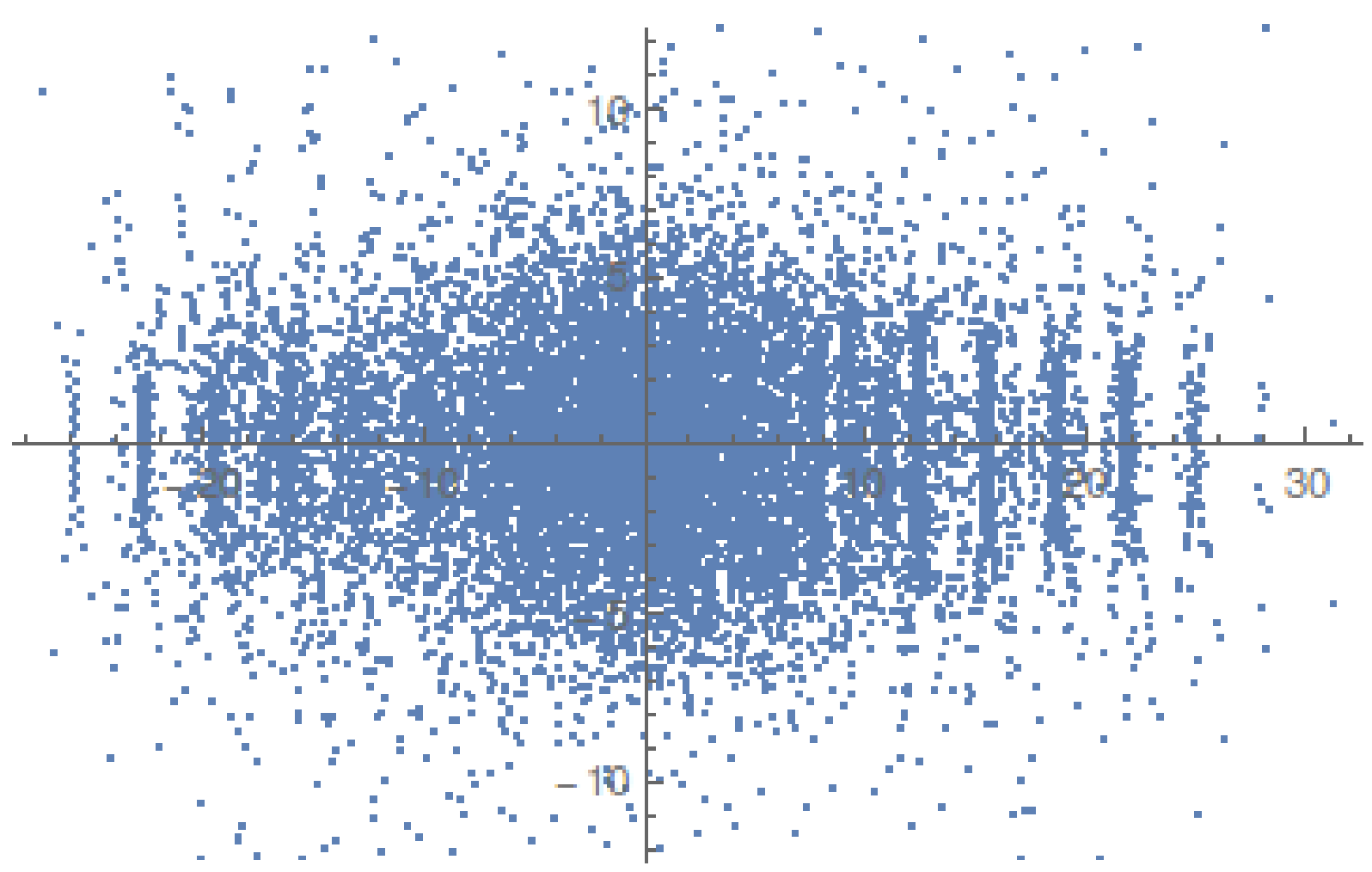

Figure 7: The Poincaré section $\left(\operatorname{Re}\left(x_{1}(t)\right), \operatorname{Re}\left(x_{3}(t)\right)\right)$ with $\operatorname{Re}\left(x_{2}(t)\right)=0$ for Sprott-A system for initial condition $\left(x_{1}(0), x_{2}(0), x_{3}(0)\right)=\left(0,5+\epsilon_{2}, 0\right)$. The time parameter runs from 0 to 20000. 